\title{
Desde I benandanti a Menocchio. Un juego de paciencia conjetural y contextual (Italia, 1966-1976)*
}

\author{
Rafael Gaune / Claudio Rolle \\ Instituto de Historia \\ Pontificia Universidad Católica de Chile
}

... me parece que el nombre de homo ludens,

el hombre que juega, expresa una

función tan esencial como la de fabricar, y merece, por lo tanto, ocupar su lugar junto al de homo faber

(Huizinga 9)

Como los borgianos bibliotecarios de Babel, que buscan libros que les dará la clave de todos los demás, oscilamos entre la ilusión de lo alcanzado y vértigo de lo inasible. En nombre de lo alcanzado queremos creer que existe un orden único que nos permitirá alcanzar de golpe el saber; en nombre de lo inasible, queremos pensar que el orden y el desorden son dos palabras que designan por igual el azar

(Perec 48)

\section{Una premisa: la experimentación como juego}

Los juegos de paciencia (Giochi di pazienza), parte del título de este ensayo, es un libro formidable y desconocido para una gran mayoría de lectores hispanoparlantes. En 1975 fue publicado por los historiadores italianos Adriano Prosperi y Carlo Ginzburg en la prestigiosa editorial Einaudi. Amigos de generación y compromiso cívico, ambos nacidos en 1939, están marcados por las lecturas de Quaderni del carcere de Antonio Gramsci y la filosofía de Benedetto Croce, así como también por la literatura de postguerra de Primo Levi, Carlo Levi, Cesare Pavese e Ítalo Calvino (Fernández García 60-77; Calvino 200-206) ${ }^{1}$ y los recorridos estudiantiles y políticos en

\footnotetext{
* Aquí se juntaron y complementaron las dos ponencias leídas en el seminario Menocchio, nuestro contemporáneo. A 40 años de El queso y los gusanos, desarrollado el 6 de octubre de 2016, organizado por el Instituto de Historia UC.

1 Existen vínculos entre Primo Levi e Ítalo Calvino a partir del término «zonas grises» (Ginzburg "Levi" 108-114).
} 
la Scuola Normale Superiore di Pisa2. Los Giochi di pazienza. Un seminario sul Beneficio di Cristo (Ginzburg y Prosperi) 3 lo redactaron en cierta forma anónima, no identificando los fragmentos escritos por cada uno, salvo por la novedosa, aunque descifrable, indicación que el «autor A» había llegado a la figura de Tullio Crispoldi mediante una investigación acerca del obispo de Verona Gian Matteo Giberti (Prosperi)4 , mientras que el «autor B» conocía a Crispoldi y sus escritos por otros autores -Ginzburg-5. Además, ofrecen otra pista de desciframiento: «uno de nosotros había tenido una formación católica»-Prosperi- (Ginzburg y Prosperi 10) ${ }^{6}$. Participaron también en el volumen los estudiantes del seminario. Antes de continuar, hacemos una advertencia. Utilizaremos los Giochi di pazienza desde el ejercicio metodológico propuesto por los autores (azar, conjeturas y falsas pistas), dejando de lado el contenido mismo del volumen?.

Decidimos redactar este ensayo a cuatro manos, no solo porque participamos en conjunto en el seminario Menocchio, nuestro contemporáneo. A 40 años de El queso y los gusanos, sino también como una forma de dialogar con un libro (escrito con varias manos) que, creemos, nos permite descifrar un contexto -Italia entre los años 1966 y 1976- y la prehistoria de

2 Una buena forma de ingresar al mundo contextual e intelectual de estos historiadores es por medio de sus propias reflexiones autobiográficas. Por ejemplo, esto se puede encontrar en el ensayo de Adriano Prosperi titulado "La mia liberazione", publicado el 25 de abril de 2016 en la revista Micromega del diario Repubblica. Podríamos incluir también la recopilación de sus escritos en diarios titulado Cause perse. Un diario civile. Para Carlo Ginzburg el ensayo "Some Queries Addressed to Myself" es una buena forma de aproximarse a su contexto intelectual e histórico.

3 Un año antes de la publicación del libro ambos publicaron el ensayo "Le due redazioni del Beneficio di Cristo. Una reseña del libro fue realizada por M. Fois en La Civiltà cattolica. Recientemente Vincenzo Vozza ha publicado un ensayo sobre el Beneficio títulado "Il Beneficio di Cristo: il 'dolce libriccino' degli 'spirituali' italiani"

4 El autor A es entonces Adriano Prosperi que, en 1969, publicó el libro Tra evangelismo e controriforma. G.M. Giberti (1495-1543).

5 El autor B es Carlo Ginzburg.

6 "Che in questo senso ci spingessero le nostre propensioni ludiche, è evidente. Ma nella stessa direzione andavano anche le nostre propensioni ideologiche più o meno latenti: tendenzialmente esse coincidevano, nonostante la diversità delle biografie (uno di noi aveva avuto una formazione cattolica)".

7 Un breve resumen de las vicisitudes de autor y editoriales del Beneficio di Cristo fue escrito, en 1980, por Maria Cali: "Todo esto ha surgido tras el reciente estudio del Beneficio de Cristo, la conocida obrita que cada vez ha ido dejando patente más y más su relación con el ambiente de [Reginald] Pole y, concretamente, de [Marco Antonio] Flaminio. No nos detendremos en los innumerables problemas que la obra ha planteado a la crítica, remitiéndonos para ello a la amplia bibliografía existente sobre el tema. El punto ya aceptado es que la composición definitiva del libro es obra de Flaminio, que reelaboró un texto compilado antes por un monje benedictino, Benedetto de Mantua, identificado como Benedetto Fontanini, que vivió hasta 1537 en el monasterio de San Giorgio Maggiore de Venecia, y de allí marchó a Catania, donde había sido destinado. Es admisible la teoría de que hiciese una parada en Nápoles en su viaje a Catania, o incluso después; la atestiguan tanto Caracciolo, que releva la conexión del benedictino con [Juan de] Valdés, como Basalú en su declaración ante el Santo Oficio. Y está documentada también su familiaridad con el círculo de Viterbo, como se aprecia en una carta enviada desde esa ciudad el 18 de julio de 1542 por Alvise Priuli a Beccadelli (...) Posteriormente, la crítica ha intentado definir los derroteros ideológicos de la obra, teniendo en cuenta que fue juzgada ya por sus contemporáneos como herética y luterana, y por lo tanto prohibida" (123). 
la obra más conocida de Carlo Ginzburg, El queso y los gusanos ${ }^{8}$, publicado en 1976 también por la editorial Einaudi. Nos permite, asimismo, «jugar» y, a diferencia de los autores de Giochi di pazienza, no descifraremos quiénes son el autor $\mathrm{A}$ y autor $\mathrm{B}$ de este ensayo, aunque podríamos decir que tanto el autor A y el autor B estudiaron su Licenciatura en Historia en la UC; realizaron estudios de postgrado en Pisa; dictan el curso Historia Moderna en el Instituto de Historia de la Pontificia Universidad Católica de Chile; tienen serias discrepancias respecto del inicio cronológico de la Edad Moderna; y el año 2016 uno de los autores le regaló Giochi di pazienza al otro. No obstante, para efectos prácticos y analíticos, es totalmente irrelevante identificarlos.

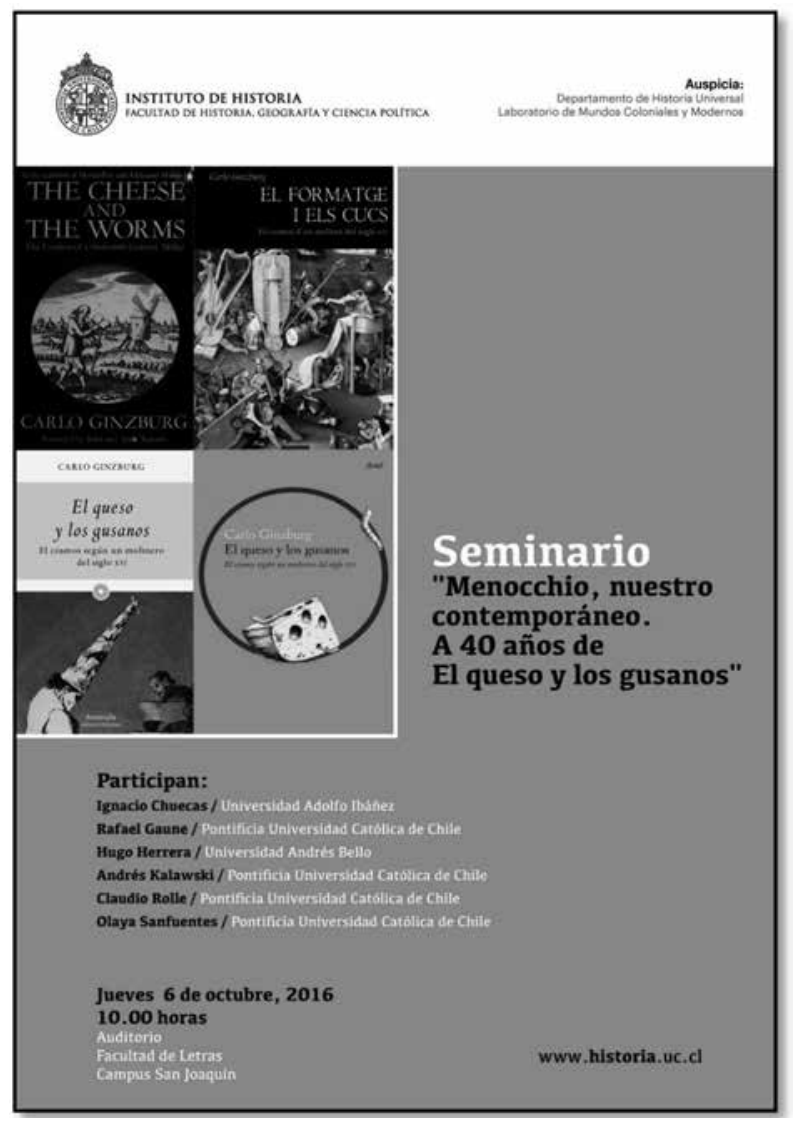

Estamos en Bolonia, 1971. Ambos historiadores, luego de su amistad juvenil pisana, se reencuentran en un seminario de investigación que deciden dictar juntos. Estudiaron en la Scuola Normale Superiore y resolvieron poner en práctica los seminarios realizados con cuatro grandes maestros como

8 Carlo Ginzburg, I formaggi e i vermi. Il cosmo di un mugnaio del '500 [1976]. Torino: Einaudi, 2009. 
el modernista Armando Saitta; Delio Cantimori9, estudioso de los herejes italianos; Arnaldo Momigliano10, uno de los clasicistas más reconocidos mundialmente hasta hoy; y el medievalista Arsenio Frugoni, precursor, en cierto sentido, de la microhistoria11 italiana con su Arnaldo da Brescia nelle fonti del secolo XII (Frugoni)12:

Habíamos estudiado juntos en Pisa; después de diez años nos habíamos encontrado, por pura casualidad, en Boloña. Enseñábamos en dos facultades distintas (letras y magisterio) a cien metros de distancia. En el otoño de 1971 decidimos reunir a nuestros estudiantes y organizar con ellos unos seminarios. En uno de esos seminarios, propusimos leer el Trattato utilissimo del beneficio di Gesú Cristo crocifisso verso i cristiani. La elección de este texto cinquecentesco, conocido solo por los especialistas de historia religiosa italiana del siglo XVI, se explicaba fácilmente. Reunía, de hecho, las siguientes condiciones: se trataba de un libro importante; proponía una serie de problemas, desde la atribución a la interpretación, solo en parte resueltos ${ }^{13}$.

Escrito y pensado entre 1971 y 1975, antes de la fama mundial de Ginzburg con su El queso y los gusanos y de la consagración de Prosperi como uno de los mayores especialistas de los estudios inquisitoriales ${ }^{14}$, el libro describe

9 De hecho, Delio Cantimori es uno de los historiadores más citado en las notas del libro. Véase el importante libro escrito por Cantimori, Eretici italiani del Cinquecento e prospettive di storia ereticale italiana del Cinquecento [1939]. Una visión de Prosperi de la historiografía de Cantimori y de su influjo, en la introducción del volumen citado en precedencia. Una revisión bibliográfica en torno a los temas investigados por Cantimori en Vincenzo Lavenia, "Bibliografia ragionata". Carlo Ginzburg escribe sobre Delio Cantimori en "Ginzburg racconta Cantimori". Un volumen que recoge documentos en torno a Cantimori en Delio Cantimori (1904-1966). Libri, documenti e immagini dai fondi della Scuola Normale Superiore.

10 Una aproximación a su obra en Peter N. Miller, Momigliano and Antiquarianism. Foundations of the Modern Cultural Sciencies.

11 Corriente historiográfica creada en Italia en la década de los 70 del siglo XX, bajo el desarrollo institucional de la editorial Einaudi y su colección "Microstorie", así como también de la revista Quaderni Storici. La premisa general de los historiadores era realizar una lectura a contrapelo de las grandes estructuras (como lo hacían los estructuralistas franceses, o bien los seguidores de la Escuela de los Annales), concentrándose en lo micro y en los casos estudios que develaban imaginarios profundos. Se puede decir que Ginzburg, entre otros, es uno de los precursores de esta forma de hacer historia. Acerca de los recorridos de la microhistoria véase Carlo Ginzburg, "Microstoria: due o tre cose che so di lei" (1994). 12 Un repertorio de los trabajos de Arsenio Frugoni en un artículo de Clara Gennaro titulado "Bibliografia degli scritti di Arsenio Frugoni".

13 "Avevamo studiato insieme a Pisa; dopo dieci anni ci eravamo ritrovati, per puro caso, a Bologna. Insegnavamo in due facoltà diverse (Lettere e Magistero) a cento metri di distanza. Nell'autunno del '71 decidemmo di riunire i nostri studenti e di organizzare con loro dei seminari. In uno di questo proponemmo di leggere Trattato utilissimo del beneficio di Gesù Cristo crocifisso verso i cristiani. La scelta di questo testo cinquecentesco, conosciuto solo dagli specialisti di storia religiosa italiana del Cinquecento, si spiegava facilmente. Esso riuniva infatti le seguenti condizioni: si trattava di un testo importante; proponeva una serie de problemi, dall'attribuzione all'interpretazione, solo in parte risolti" (Ginzburg y Prosperi 3). 14 Sin duda, su libro mayor concerniente a la Inquisición romana es Tribunali della coscienza. Inquisitori, confessori, missionari [1996] que, en cierto modo, cierra con el diccionario de 
el desarrollo del seminario desde el primer día hasta el final del semestre. Ese primer día entregaron a los estudiantes el libro del Cinquecento italiano titulado Trattato utilissimo del beneficio di Gesú Cristo crocifisso verso i cristiani (Ginzburg y Prosperi 215-258). Estupefactos, los estudiantes debían descubrir al posible autor, las ediciones, comparar ediciones y fragmentos de otros textos, la prehistoria del texto y el significado del libro por medio de un intenso trabajo textual y filológico crítico. En otras palabras, descubrir y descifrar un libro conocido solo por especialistas de la historia de la religión en contexto reformista, sumamente erudito y con una gran fortuna en el siglo XVI. Era un trabajo colectivo con estudiantes, como expresan en la introducción, mediante un trabajo lúdico y artesanal y, por ningún motivo, buscando el placer de la investigación como privilegio exclusivo del director del equipo, como escriben Ginzburg y Prosperi, en modo crítico, respecto de los trabajos colectivos programados por Annales (Ginzburg y Prosperi 10) ${ }^{15}$.

Este libro siempre lo hemos comparado metafóricamente con el viaje en 1968 de The Beatles a la India, o bien como una pieza experimental del neorrealismo italiano, sencillamente porque comparten la búsqueda de la experimentación. Es un texto de pura experimentación metodológica. De una búsqueda constante por encontrar documentos y ponerlos a prueba. De interrogarlos y diseminarlos. Es un libro que en estos tiempos marcados por la indexación y las revistas con «impacto», sería imposible publicar. Solo un pequeño ejemplo de la experimentación a la que nos referimos, se encuentra en el párrafo inicial del libro:

No se necesita llevar la cocina a la mesa, advertía en alguna parte lord Acton. Hemos buscado transgredir lo máximo posible este precepto de etiqueta historiográfica. En cambio, de un pollo asado con papas fritas, el lector encontrará un pollo vivo revoloteando, provisto de plumas y con cresta. Más allá de esta metáfora, no es una investigación acabada y completa, sino se encuentran los vaivenes de la investigación, las pistas falsas seguidas y descartadas antes de llegar al resultado considerado aceptable. Deseamos que todo esto no resulte demasiado indigesto (Ginzburg y Prosperi 3) ${ }^{16}$.

\footnotetext{
la inquisición coordinado por él: Dizionario storico dell'Inquisizione, coordinado por Adriano Prosperi, con la colaboración de Vincenzo Lavenia y John Tedeschi. Una reseña de los Tribunali, escrita por Carlo Ginzburg, titulada "Italia, un paese fondato sull'Inquisizione" en La Repubblica (14 de enero de 1997).

15 Acerca de este punto escriben lo siguiente: "Ma il piacere della ricerca è legato a una situazione artigianale, destinata a sparire quando la (forse inevitabile) parcellizzazione industriale del lavoro penetra nell'organizzazione degli studi storici. Nei grandi lavori collettivi, come per esempio quelli programmati dal gruppo Annales, il piacere della ricerca diventa privilegio quasi esclusivo del direttore dell'équipe, che prima propone gli obiettivi del lavoro ripetitivo eseguito dai subordinati (uomini e macchine) e poi ne interpreta i risultati".

16 "Non bisogna portare la cucina in tavola. Ammoniva da qualche parte lord Acton. Abbiamo cercato di trasgredire il più possibile questo precetto di etichetta storiografica. Anziché un pollo arrosto con contorno di patate fritte il lettore si troverà sul piatto un pollo vivo e starnazzante, provvisto di penne e bargigli; fuor di metafora, non una ricerca rifinita e compiuta ma gli andirivieni della ricerca, le false piste seguite e scartate prima di arrivare al risultato ritenuto accettabile. Ci auguriamo che tutto ciò non risulti troppo indigesto".
} 
Es un texto escrito «a fuego lento», condicionado por una lectura lenta, la incertidumbre y las inquietudes audaces y vivaces de los estudiantes; a contrapelo de una idea de producción científica contemporánea y los caprichos de la indexación. Un libro profundamente sarcástico y autoirónico que termina de esta forma -luego de 188 páginas de ensayo metodológico, y antes de colocar el Beneficio di Cristo como apéndice desde la página 215 a la 258- «comencemos a escribir».

Los juegos de paciencia están llenos de intentos fallidos, incertidumbres, caminos de investigación propuestos por los estudiantes y pasos en falso que son descritos sin filtros por los historiadores. El libro no llega a ninguna conclusión, pero nos da una gran lección de metodología. No tiene pretensiones de sistematicidad sino, al contrario, se mueve en el plano de las potencialidades de las conjeturas. Y eso, el libro, lo hace explícito. Ginzburg, en los noventa, declaró que el libro era un tanto oscuro e «ininteligible». Tiene un poco de razón. Es difícil leerlo. Incluso traducirlo y explicarlo en un contexto no italiano. Pero las enseñanzas quedan. El libro funciona en ellos como retrospectiva y prospectiva que marca, en cierto sentido, el modo en que entienden hasta hoy la investigación histórica: como un verdadero juego de paciencia entre el presente y el pasado, entre los muertos y los vivos, entre los documentos y sus inquisidores. Antes de descifrar la prehistoria de El queso y los gusanos a partir de Giochi di pazienza, presentaremos el paisaje intelectual de Italia en las décadas de los 60 y 70 del siglo XX que es, finalmente, donde llega y luego renace Domenico Scandella: el molinero Menocchio.

\section{¿A qué mundo llega Domenico Scandella? ¿Cómo se produce la resurrección de Menocchio?}

Estas dos preguntas nos han venido dando vueltas en la cabeza desde que decidimos «celebrar el cumpleaños» de Domenico Scandella -conocido mundialmente como Menocchio-, el jueves 6 de octubre de 2016 a las 10:00 horas en el Auditorio de la Facultad de Letras de la Pontificia Universidad Católica de Chile. Respecto de la palabra «celebrar», no obstante, el autor A y autor B de este ensayo, nunca llegaron a un acuerdo. Uno decía que no se podía «celebrar» la condena y muerte de Menocchio, mientras que el otro planteaba «celebrar» el renacimiento. Que el seminario se haya titulado Menocchio, nuestro contemporáneo, y no Feliz cumpleaños, Menocchio, representó, en cierto sentido, un punto de convergencia entre ambos autores de este ensayo.

Esa discrepancia, sin embargo, nos permitió acordarnos de Michel de Certeau y sus palabras iniciales en "La operación historiográfica", en su libro L'écriture de I'histoire (1975), donde pregunta y reflexiona acerca de la relación enigmática que se establece entre el presente y la muerte gracias a la mediación de unas actividades técnicas. Acerca de esas actividades técnicas que lo sacan del archivo y de las formas de representar el mundo invitándolo a escuchar -como decía en su momento Jacques Attali- a una cultura popular mucho más compleja y dinámica de lo que se presentaba frecuentemente 
(Ginzburg Il formaggio XII) ${ }^{17}$. Con mirada crítica y un deseo de autonomía en el modo de abordar las fuentes -esas fuentes orales donde la circularidad y modelación están presentes-, Ginzburg apunta al rescate de las voces que nos llegan del pasado, con respeto y con la atención necesaria para evitar imponer a los habitantes de ese país del pasado, categorías e ideas fijas y definidas cuando no prejuicios y combates anacrónicos. Esta escucha del autor, que presenta su libro a los 37 años será, en nuestra opinión, uno de los rasgos distintivos de esta obra, una suerte de retrato de oídas, de retrato hablado y uno de los motivos del porqué El queso y los gusanos alcanza un éxito significativo desde su aparición hasta el presente.

Carlo Ginzburg estaba preparado, como se podrá leer en los próximos párrafos, para realizar o intentar realizar una de las mayores tareas del oficio de la historia, la más compleja y ambiciosa, la más hermosa y generosa: la tarea de devolver vida a los muertos, la de resucitar a quienes ya habían pasado de este mundo, dándoles la posibilidad de que se instalen entre nosotros y se vuelvan nuestros contemporáneos, ofreciéndoles una nueva posibilidad de hablar y contar, ofreciéndonos la oportunidad de escuchar y preguntar con una curiosidad cargada de cuatro siglos de eso que llamamos historia.

Ginzburg es un buen intérprete de su mundo, de su tiempo y espacio, y por ello no está solo en estos esfuerzos. Queremos recordar que, en estos mismos años de investigación y estudio, el inglés Peter Burke trabajaba para presentar, en 1978, su Cultura popular en la Europa moderna que, parafraseando un título de película, está «tan lejos tan cerca» del espacio transitado en la primera mitad de la década por Carlo Ginzburg. Ese año de 1978 será también el del estreno de L'albero degli zoccoli18, el film de Ermanno Olmi, girado en el año anterior y que puede dialogar con naturalidad con la historia de Menocchio y sus contemporáneos de esos años en que Domenico Scandella era un molinero del Friuli y no un ciudadano del mundo, un habitante de la historiografía. Tanto el historiador inglés como el cineasta bergamasco estaban en la línea que Ginzburg había marcado con intensidad y originalidad al buscar recorrer esa distancia temporal y cultural que mediaba entre los tiempos de los juicios a Menocchio, por un lado y la Italia y el mundo de los años setenta del siglo XX, por el otro.

Para recorrer esa distancia Ginzburg se prepara con atención y diríamos cautela, consciente de que las palabras y la oralidad tienen un relieve muy complejo y que el oficio de traductor es riesgoso pero, a pesar de todo, hermoso y generoso (Kula 234) ${ }^{19}$.

\footnotetext{
17 Acerca de este punto, Ginzburg escribe: «L'esistenza di dislivelli culturali all'interno delle cosiddette società civilizzate è il presupposto della disciplina che si è autodefinita via via folklore, demologia, storia delle tradizioni popolari, etnologia europea. Ma l'uso del termine 'cultura' per definire il complesso di atteggiamenti, credenze, codici di comportamento e così via, propri delle classi subalterne in un dato periodo storico, è relativamente tradivo, e mutato dall'antropologia culturale».

18 Película que gira en torno a la historia de la vida cotidiana de un grupo de campesinos de la región de Lombardía a fines del siglo XIX. La película incluye campesinos locales en vez de actores profesionales y fue grabada en bergamasco, el dialecto de la zona.

19 Respecto del historiador como traductor, Witold Kula ofrece una preciosa definición: "El historiador debe ser un intérprete que traduce los valores de otras civilizaciones a nuestra
} 
Esta preparación no solo pasa por la adecuación de los sentidos para percibir un mundo ajeno y próximo a la vez; no solo para mirar y ver, para escuchar palabras y silencios, para percibir el peso de las mediaciones de toda índole -en especial de los inquisidores y sus traducciones de lenguajes e imágenes populares-, sino de manera muy significativa pasa también por su escritura, por su capacidad de dar cuenta de lo vivido de regreso ya no del Chad, no del Congo o de la Unión Soviética, como hiciera en su momento con sus libros de testimonio y denuncia André Gide, si no de regreso del Friuli de los años de la Reforma y sus consecuencias, de sus juicios y sospechas, de sus perplejidades y temores.

Hay en la escritura de El queso y los gusanos un cuidado particular por la tarea de comunicar, con una muy especial atención al lector, con una consciencia clara y vigorosa de que se puede y se debe dar una triangulación entre el resucitado Menocchio, el taumaturgo Ginzburg y el a veces llamado «generoso» otras «gentil» o «curioso» lector. Es una suerte de diálogo para llegar a comprender un drama complejo y excepcional que a la vez puede servir para una comprensión más amplia de un mundo que hemos perdido. Todo esto empalma, asimismo, con un tiempo que el historiador inglés Paul Ginsborg ha llamado «la época de la acción colectiva», refiriéndose a fines de los sesenta e inicios de los setenta del siglo XX; el tiempo en que comienza la aventura de la dupla Menocchio-Ginzburg. Escribe, Ginsborg:

Desde 1968 en adelante la inercia de los dirigentes fue sustituida por la actividad de las bases, dando origen a la más grande estación de acción colectiva en la vida de la República. El movimiento italiano fue el más profundo y duradero de ese año 1968 europeo (404).

Es el espíritu crítico del 68 con su mirada inquieta y sus anhelos amplios los que marcarán a la generación de Carlo Ginzburg. La reforma de la educación y la universidad, el deseo de cambiar el mundo con una premura que se manifiesta en la vida política y social y movimientos como «Servire il popolo», «Avanguardia Operaia», «Potere operaio», «Lotta continua», o «Il Manifesto», dejarán una huella en el historiador. Son años acelerados y maximalistas en sus demandas y en sus modos de actuar, cuestionando el orden político preexistente y alcanzando niveles de debate ácidos y de dureza inusitada y que comprende desde los duros términos con que Pier Paolo Pasolini se refiere a los estudiantes movilizados y sus modos de proceder, hasta la radicalización, la crisis y el inicio de lo que se ha llamado en jerga italiana «los años de Plomo»: violencia política que se extiende sostenidamente con presencia de grupos radicales que optan por el terrorismo y el resurgimiento del fascismo de izquierda y de derecha. Se trata de un período particularmente desafiante para las estructuras políticas, económicas, sociales y culturales de Italia que son cuestionadas y tensionadas de diversas formas: es el período no solo

lengua. Él estará siempre consciente de los valores individuales que traduce, como también de la posibilidad, a pesar de todo, de realizar dicha traducción. El historiador hace a la sociedad consciente de la propia individualidad y al mismo tiempo hace esta individualidad comprensible a los otros. Comprender a los otros, esta es la tarea que el historiador debe prefijarse. No es fácil tener una más difícil. Es difícil tener una más bella". 
de los grandes movimientos estudiantiles, sino también del resurgimiento del maximalismo revolucionario, de las manifestaciones por el derecho a la vivienda digna, de constantes movilizaciones sindicales y ciclos de huelga con aspiraciones amplias y ambiciosas, de desafíos a la primacía de la DC y del debate pertinente al divorcio. En tiempos del «otoño caliente», para usar un término de la época, Ginzburg transita entre este mundo agitado y el de los tiempos de la Reforma protestante, de la Contrarreforma, de la Reforma Católica, en una Italia llena de procesos, de pugnas entre ortodoxias y herejías, donde la muerte por causa de las ideas opera con la Inquisición, así como conoció el propio Domenico Scandella que, cruzándose accidentalmente con Ginzburg, encontró a su liberador al cabo de varios siglos. El historiador y el molinero comenzaron una amistad destinada a durar. Retornemos, ahora, a los juegos de paciencia que vincula paisaje intelectual italiano del siglo XX y las inquietudes metodológicas de Ginzburg y Prosperi.

\section{La experimentación como paciencia}

Giochi di pazienza fue publicado en 1975, como indicamos, un año antes de El queso y los gusanos. Este dato es relevante para entender cómo Carlo Ginzburg aborda y reconstruye la vida de Menocchio. Aunque es un vínculo que puede ser obvio, estos dos textos imprimen la forma de cómo Ginzburg entiende la historia y la historiografía, marcando la reflexión e introspección metodológica que en paralelo realizaba con la reconstrucción de la vida de Menocchio. No podemos olvidar, en ese sentido, la tesis de doctorado de Ginzburg, defendida en Pisa en 1964 y publicada en 1966, en donde ya se había introducido en el estudio de las creencias populares de la cultura campesina por medio de expedientes de brujería de la ciudad de Módena. I benandanti20, libro producto de esa investigación, nos habla de dos aproximaciones e influjos que están hasta hoy en la producción del italiano: las influencias del Instituto Warburg de Londres con Ernst Gombrich ${ }^{21}$ y el gran cazador de mundos mágicos, el etnólogo y antropólogo cultural Ernesto de Martino22. Esto, sin duda, está latente en el Ginzburg de El queso y los gusanos y es una clave para comprender cómo absorbe la humanidad y los recorridos biográficos del condenado Menocchio. Así como Ginzburg descifró a Menocchio no solo por medio de las actas de los procesos inquisitoriales, sino también por sus reinterpretaciones de lecturas, como, por ejemplo, el Decamerón de Giovanni Bocaccio, Florilegio de la Biblia, Il cavallier Zuanne,

\footnotetext{
20 Para una reflexión de este libro y su posterior amplificación investigativa, véase el ensayo escrito por Carlo Ginzburg en este dossier de Taller de Letras, "Los benandanti, cincuenta años después".

21 Las preocupaciones de Ginzburg sobre Gombrich ya habían sido expuestas por él en una monografía publicada el mismo año que $I$ benandanti: Da A. Warburg a E. H. Gombrich. Note su un problema di metodo. Esto retorna, constantemente, en la obra de Ginzburg, como, por ejemplo, por medio del uso del término «Pathosformel» de Aby Warburg en su libro Paura reverenza terrore. Cinque saggi di iconografia política.

22 Ernesto de Martino, antropólogo italiano (1908-1965), destacado por sus importantes contribuciones a los estudios religiosos del sur de Italia, publicó en 1948 su fundamental libro Il mondo magico: prolegomeni a una storia del magismo y en 1961 La terra del rimorso. Contributo a una storia religiosa del Sud. Ginzburg publicó un ensayo sobre de Martino titulado "Momigliano and De Martino". Una reciente biografía enfatiza los aportes de la obra de de Martino: Giordana Charuty, Ernesto de Martino. Les vies antérieures d'un anthropologue.
} 
Historia del Giudicio, el Corán e Il Sogno di Caravia, una forma de descifrar al historiador italiano es analizando Giochi di pazienza como una prehistoria de El queso y los gusanos.

Ginzburg se encontraba en su fase de experimentación y búsqueda filológica, palabra por palabra, buscando las anomalías como lo hiciera Roman Jakobson, viajando y haciendo un tour archivístico por Italia y siguiendo al pie de la letra la propuesta del filólogo italiano Carlo Dionisotti quien, en 1968, formuló un programa de investigación mediante la siguiente frase: «por mero azar, es decir por la norma que preside a la investigación»23. Escribe, Ginzburg, de esto el 2001, vinculando sus pasadas investigaciones con sus actuales preocupaciones metodológicas:

'Por mero azar, es decir por la norma que preside a la investigación de lo desconocido': hace mucho tiempo estas palabras de Carlo Dionisotti nos parecieron a Adriano Prosperi y al que esto escribe convenientes para introducir, algunas consideraciones sobre la imbricación entre caso y presupuestos (ideológicos o de otro tipo) dentro de la investigación histórica (Ginzburg "Conversar" 229).

En esa búsqueda de «leer lentamente» las fuentes y sus palabras, como propone Nietzsche (otro filólogo, por cierto) en el prólogo de Aurora24, elaboró su «paradigma indiciario» para su célebre ensayo «Huellas. Raíces de un paradigma indiciario» 25 , publicado en 1979 en un volumen colectivo -fuertemente pisano-, titulado Crisi della ragione y editado por el filósofo analítico Aldo Giorgio Gargani. Asimismo, mientras Prosperi y Ginzburg comenzaban el seminario en Bolonia sobre El beneficio de Cristo, en 1972, en el primer tomo de la Storia d'Italia de la editorial Einaudi, Carlo Ginzburg desplegó como una verdadera pista de investigación -en forma vanguardista y pionera el vínculo de las «indias de por acá» (el exótico europeo) con la óptica misionera mundial (expansión global de las conversiones religiosas). En el sugerente ensayo, «Folklore, magia, religione», lleno de matices y propuestas de investigación, analizó esa fórmula lingüística que emerge de los documentos jesuíticos del siglo XVII y la cruzó con dos planos y registros que se entrelazaron e interactuaron con la evangelización en Asia y las Américas 26.

\footnotetext{
23 Frase escrita por Dionisotti, como un verdadero programa de investigación, en "Resoconto di una ricerca interrotta" (Ginzburg y Prosperi 125). Ginzburg, posteriormente, y partiendo de esta frase, escribe "Conversare con Orion", en donde reflexiona también sobre Giochi di pazienza. En español el ensayo se encuentra en el libro Tentativas. Recientemente Michele Lodone ha rastreado la frase de Dionisotti en "Campana Dionisotti Cantimori. Intorno a una interrotta ricerca a tre".

24 Aparte de «leer lentamente» Nietzsche dice que hay que "mantenerse aparte, darse tiempo, hacerse silencioso, hacerse lento" (16).

25 Ítalo Calvino escribió un ensayo sobre las implicancias analíticas de este paradigma titulado "L'orecchio, il cacciatore, il pettegolo", en La Repubblica (20 de enero de 1980). 26 "Ma ciò che rese ancora più significativa quest'opera di cattolicizzazione fu il fatto che essa fosse rivolta, soprattutto grazie ai gesuiti, in direzione del Mezzogiorno. La frase, tanto spesso citata, sulle 'Indie di quaggiù' -e cioè I'Italia meridionale- non era un'espressione retorica. Nel momento in cui la Chiesa assumeva, non senza contraddizioni, un'ottica
} 
Esta pista de investigación, Ginzburg, nunca más la desarrolló27. Sin embargo, esos planos históricos entrecruzados fueron sistematizados y profundizados por Adriano Prosperi, su compañero de juegos de paciencia, en un seminario desarrollado en Florencia en 1980, con un sugerente e influyente texto «Otras indias: missionari della Controriforma tra contadini e selvaggi» $y$, posteriormente, en su ensayo «Il missionario» 28 , en el que examinó el tránsito de los misioneros jesuitas Silvestro Landini y Cristóbal Rodríguez. El primero, se refirió a la Córcega como «mi india», en Bastia el 7 de febrero de 1553. El segundo, por su parte, el 27 de abril de 1556 en Gandía, estudió los problemas de cristianización de los «moriscos» en un plano comparado con las Indias. Ambos, sin duda, se movieron en sus «propias indias» imaginarias y reales al interior de los márgenes europeos. Tanto Prosperi como Ginzburg, por medio de inspiradores y densos ensayos, abrieron un camino de pesquisa que marcó a las historiografías de las misiones, sobre todo italiana y francesa, en las décadas de los años 80 y 90 del siglo $X^{29}$.

Así, podríamos enunciar que, I benandanti, Giochi di pazienza y «Folklore, magia, religione», es la tríada metodológica que precede la restitución de la humanidad de Menocchio, incluyendo, por cierto, el paisaje intelectual italiano entre 1966 y 1976 . Esa restitución, ese restablecimiento, es el «retoñar», como escribe el poeta Miguel Hernández en «El herido»: «retoñarán aladas de savia sin otoño/reliquias de mi cuerpo que pierdo en cada herida. /Porque soy como el árbol talado, que retoño: porque aún tengo la vida» (Hernández). Una vida como la de Menocchio, talada por la inquisición, «retoña» en 1976 porque aún tenía vida.

El hereje del Friuli no surge de la nada. No solo emerge porque Ginzburg «por mero azar» encontró materialmente el rico y oscuro expediente del proceso inquisitorial del molinero, sino emerge porque tuvo la paciencia de «leerlo lentamente», palabra por palabra, indicio por indicio30. Todo lo

missionaria mondiale, la penetrazione religiosa nelle campagne dell'Italia meridionale si configurava come un corrispettivo, su un altro piano, delle iniziative di evangelizzazione in Asia o nelle Americhe" (Ginzburg "Folklore" 656-657)

27 Una aproximación similar puede leerse en Carlo Ginzburg, "Le voci dell'altro. Una rivolta indigena nelle Isole Marianne".

28 "Colui che è spesso ricordato come il primo, più consapevole e più appassionato missionario delle 'Indie di qua', inizio la sua carriera nelle montagne della Garfagnana andando a caccia di eretici e scontrandosi col clero locale su questioni come la grazia e il libero arbitrio. E in Spagna, dove non ci fu una penetrazione significativa della Riforma, il problema dominante fu quello delle minoranze maomettane da convertire: infatti, tra i primi a parlare di 'otras Indias' fu il padre Cristoforo Rodríguez, preoccupato per le difficoltà delle conversioni dei 'moriscos'. La nozione e l'immagine delle Indie serviva di buon auspicio, implicando una facile e larga messe da raccogliere secondo gli stereotipi della cultura missionaria dell'epoca; e confortava chi si sentiva impiegato in compiti minori rispetto all'ideale apostolico che lo muoveva verso le terre lontane" (Prosperi 204)

29 Un recorrido historiográfico de esas premisas en el ensayo de Rafael Gaune titulado "Nuestra casa es el mundo. Historiografía y registros entre dos escalas: una cantera abierta". 30 Esta reflexión da cuenta de un ideal historiográfico que Ginzburg, en 1980, profundizando y polemizando en torno a su "paradigma indiciario" propone en estos términos: "mi ideal historiográfico sería una historiografía que fuese al mismo tiempo de tipo de Cézanne, pero también de tipo de Monet, es decir, que nos diese también la fragilidad de lo vivido, de eso vivido que está allí y que se nos escapa, porque no cuenta para nada o casi, o porque solo 
contrario, por ejemplo -como el mismo Ginzburg advierte en la introducción de El queso y los gusanos-, de lo que hizo Michel Foucault con los expedientes del parricida Pierre Rivière (Ginzburg Il formaggio XVI-XVII)31. Fue un juego de paciencia, ahora en solitario, que transformó el espíritu de los tiempos marcados por el estructuralismo de Claude Levi-Strauss, el auge foucaultiano y la expansión de la tercera generación de Annales ${ }^{32}$.

No queda más que seguir descifrando a Menocchio, nuestro contemporáneo, y al historiador italiano, un omnívoro y taumaturgo por excelencia como se refleja en este dossier. Y, como el mismo El queso y los gusanos concluye, «De Menocchio sabemos muchas cosas. De este Marcato, o Marco -y de tantos otros como él, que vivieron y murieron sin dejar huellas- no sabemos nada» (Ginzburg Il formaggio 148).

\section{Obras citadas}

Burke, Peter. La revolución historiográfica francesa. La Escuela de los Annales: 1929-1989. Barcelona: Editorial Gedisa, 1993.

. Popular Culture in Early Modern Europe [1978]. Londres: Ashgate, 2009.

Calì, Maria. De Miguel Ángel al El Escorial. Madrid: Akal, 1994. [En italiano Da Michelangelo all'Escorial. Momenti del dibattito religioso nell'arte del Cinquecento. Torino: Einaudi, 1980]

Calvino, Italo. "Carlo Ginzburg, Espías: raíces de un paradigma indiciario". Mundo escrito y mundo no escrito [2002]. Madrid: Siruela, 2006, 200-206

"L'orecchio, il cacciatore, il pettegolo", en La Repubblica (Roma, Italia). 20 de enero de 1980.

Cantimori, Delio. Eretici italiani del Cinquecento e prospettive di storia ereticale italiana del Cinquecento [1939]. Editado por Adriano Prosperi. Torino: Einaudi, 2009.

Certeau, Michel de. L'écriture de I'histoire [1975]. Paris: Gallimard, 2002.

cuenta cabalmente para aquel que lo ha vivido. Una historiografía que entonces acertara lo mismo a reconstruir lo efímero, ese carácter efímero de lo vivido, que la geología profunda en la que esto efímero se inserta. Conozco un historiador que ha avanzado, en mi opinión, en esta justa dirección, y ese historiador es Marc Bloch" (Ginzburg "Intervención" 121). 31 Escribe Ginzburg sobre el modo en que Foucault aborda a Pierre Rivière: "Ciò che interessa soprattutto a Foucault sono il gesto e i criteri dell'esclusione: gli esclusi, un po' meno (...) Il rapporto, oscuro e contradittorio, di Pierre Rivière con la cultura dominante, è appena accennato; le sue letture (almanacchi, libri di pietà, ma anche Le bons sens du curé Meslier) sono addirittura ignorate. Si preferisce descriverlo, vagante nei boschi dopo il delitto, come 'un uomo senza cultura... un animale senza istinto... un essere mitico, un essere mostruoso che è impossibile definire perché estraneo a qualsiasi ordine enunciabile. Ci si estasia di fronte a un'estraneità assoluta che in realtà è frutto del rifiuto dell'analisi e dell'interpretazione (...) un discorso che passa attraverso il delitto e l'antropofagia, che s'incarna indifferentemente nella memoria redatta da Pierre Rivière o nel suo matricidio. È un populismo di segno rovesciato, un populismo 'nero', ma pur sempre populismo".

32 Sobre los matices en las generaciones de los Annales véase Peter Burke, La revolución historiográfica francesa. La Escuela de los Annales: 1929-1989. Los Annales en un desarrollo cronológico más amplio en Sonia Corcuera de Mancera, Voces y silencios en la historia. Siglos $X I X$ y $X X$. Georg Iggers, por su parte, realiza un interesante ejercicio al insertar a los Annales como bisagra entre la "objetividad científica" y el "desafío posmoderno" en $L a$ historiografía del siglo XX. Desde la objetividad científica al desafío posmoderno. 
Charuty, Giordana. Ernesto De Martino. Les vies antérieures d'un anthropologue. Marseilles: Éditions Parenthèses-Éditions de la MMSH, 2009.

Corcuera de Mancera, Sonia. Voces y silencios en la historia. Siglos XIX y XX. México: Fondo de Cultura Económica, 1997.

De Marino, Ernesto. Il mondo magico: prolegomeni a una storia del magismo. Torino: Einaudi, 1948.

- La terra del rimorso. Contributo a una storia religiosa del Sud. Milano: Il Saggiatore, 1961.

Dionisotti, Carlo. "Resoconto di una ricerca interrotta". Annali della Scuola Normale Superiore di Pisa 2: 37(1968): 259.

Fernández García, Blanca. "Alì Babà. Arqueología y literatura en la modernidad". Impossibilia 6. 2013: 60-77.

Fois, M. "Rassegña a Giochi di pazienza". La Civiltà cattolica 129:3, quaderno 3073. 1978: 310-311.

Frugoni, Arsenio. Arnaldo da Brescia nelle fonti del secolo XII [1954]. Torino: Einaudi, 1989.

Gargano, Aldo Giorgio, editor. Crisi della ragione. Nuovi modelli nel rapporto tra sapere e attività umane. Torino: Einaudi, 1979. [En español: Crisis de la razón. México: Siglo XXI Editores, 1983].

Gaune, Rafael. "Nuestra casa es el mundo. Historiografía y registros entre dos escalas: una cantera abierta". Escritura y salvación. Cultura misionera jesuita en tiempos de Anganamón, siglo XVII. Santiago, Ediciones Universidad Alberto Hurtado, 2016. 60-80.

Gennaro, Clara. "Bibliografia degli scritti di Arsenio Frugoni". Annali della Scuola Normale Supeiore di Pisa 3. III (1973): 487-514.

Ginsborg, Paul. Storia d'Italia dal dopoguerra a oggi. Torino: Einaudi, 2009.

Ginzburg, Carlo; Prosperi, Adriano. "Le due redazioni del Beneficio di Cristo". Eresia e riforma nell'Italia del Cinquecento: Miscellanea 1 del Corpus Riformatorum Italicorum. Firenze: Sansoni, 1974. 135-204.

Giochi di pazienza. Un seminario sul Beneficio di Cristo. Torino: Einaudi, 1975.

Ginzburg, Carlo. "Levi, Calvino et la zone grise". Témoigner. Entre histoire et mémoire 119. 2014: 108-114.

"Some Queries Addressed to Myself". Cromohs. Cyber Review of Modern Historiography. 18. 2013: 90-96.

Da A. Warburg a E. H. Gombrich. Note su un problema di metodo. Spoleto: Centro Italiano di Studi sull'Alto Medio Evo, 1966.

I formaggi e i vermi. Il cosmo di un mugnaio del '500 [1976]. Torino: Einaudi, 2009.

"Conversare con Orion". Quaderni storici 36 (2001): 905-913. [traducción al español en Tentativas. Rosario: Prohistoria, 2004. 229-239) "Le voci dell'altro. Una rivolta indigena nelle Isole Marianne". Rapporti di forza. Storia retorica prova. Milano: Feltrinelli, 2000. 87-108.

"Microstoria: due o tre cose che so di lei". Il filo e le tracce. Vero, falso, finto. Milano: Feltrinelli, 2006. 241-269.

"Momigliano and De Martino". History and Theory 30 (1991): 37-48. "Spie. Radici di un paradigma indiziario" [1979]. Miti emblemi spie. Morfologia e storia. Torino: Einaudi, 2010 [En español en Tentativas. Rosario: Prohistoria, 2004. 69-113].

"Intervención sobre el paradigma indiciario". Tentativas. Rosario: Prohistoria, 2004. 115-127. 
I benandanti. Ricerche sulla stregoneria e sui culti agrari tra Cinquecento e Seicento [1966]. Torino: Einaudi, 1966.

Paura reverenza terrore. Cinque saggi di iconografia politica. Milano: Adelphi, 2015.

Ginzburg, Carlo, "Folklore, magia, religione". Storia d'Italia. I Caratteri originali. Torino: Einaudi, 1972. 656-657.

Hernández, Miguel. El hombre acecha [1937-1939]. Obra completa 1. Barcelona: Espasa, 2010.

Huizinga, Johan. Homo ludens [1938]. Madrid: Alianza, 2012.

Iggers, Georg. La historiografía del siglo XX. Desde la objetividad científica al desafío posmoderno. Santiago: Fondo de Cultura Económica, 2012.

Kula, Witold. Riflessioni sulla storia. Venezia: Marsilio Editore, 1990.

Lavenia, Vincenzo. "Bibliografia ragionata". Delio Cantimori. Eretici italiani del Cinquecento e prospettive di storia ereticale italiana del Cinquecento [1939]. Editado por Adriano Prosperi. Torino: Einaudi, 2009.

Lodone, Michele. "Campana Dionisotti Cantimori. Intorno a una interrotta ricerca a tre". Studi Romagnoli LVI (2015): 605-622.

Menozzi, Daniele y Torchiani, Francesco, editores. Delio Cantimori. 1904-1966. Libri, documenti e immagini dai fondi della Scuola Normale Superiore. Pisa: Edizoni della Normale, 2016.

Miller, Peter N. Momigliano and Antiquarianism. Foundations of the Modern Cultural Sciencies. Toronto: Toronto University Press and UCLA, 2007.

Nietzsche, Friedrich. "Aurora". Obras completas II. Buenos Aires: Aguilar, 1967.

Perec, Georges. Pensar / Clasificar [1985]. Barcelona: Gedisa, 2007.

Pozzi, Regina, Prosperi, Adriano, editores. Studi in onore di Armando Saitta dei suoi allievi pisani. Pisa: Giardini, 1989.

Prosperi, Adriano. "Otras indias: missionari della Controriforma tra contadini e selvaggi". Garfagnini, Giancarlo, editor. Scienze, credenze occulte, livelli di cultura. Firenze: Leo S. Olschki, 1982. 205-234.

Prosperi, Adriano. "La mia liberazione". Rivista Micromega. [Roma, Italia]. 25 abr. 2016. [url: http://temi. repubblica. it/micromega-online/ adriano-prosperi-la-"mia"-liberazione-2/].

Cause perse. Un diario civile. Torino: Einaudi, 2010.

Tra evangelismo e controriforma. G. M. Giberti. 1495-1543. Roma:

Edizioni di Storia e Letteratura, 1969.

Tribunali della coscienza. Inquisitori, confessori, missionari [1996].

Torino: Einaudi, 2009.

"Il missionario". Villari, Rosario, coordinador. L'uomo barocco [1991]. Roma-Bari: Editori Laterza, 2005.

Stabile, Alberto. "Carlo Ginzburg racconta Cantimori". I buoni maestri. Milano: Mondadori, 1988

Vozza, Vincenzo. "Il Beneficio di Cristo: il 'dolce libriccino' degli 'spirituali' italiani". Eretici, dissidenti, inquisitori. Per un dizionario storico mediterraneo. Roma: Aracne editrice, 2006. 77-86. 